\title{
A research update on the anticancer effects of bufalin and its derivatives (Review)
}

\author{
YU-LONG LAN ${ }^{1-3^{*}}$, JIA-CHENG LOU ${ }^{1,2^{*}}$, XUE-WEN JIANG ${ }^{1,2^{*}}$, XUN WANG $^{1,2}$, \\ JIN-SHAN XING ${ }^{1,2}$, SHAO LI $^{3}$ and BO ZHANG ${ }^{1,2}$ \\ ${ }^{1}$ Department of Neurosurgery, The Second Affiliated Hospital of Dalian Medical University, Dalian, \\ Liaoning 116023; ${ }^{2}$ Department of Neurosurgery, Shenzhen People's Hospital, Shenzhen, \\ Guangdong 518020; ${ }^{3}$ Liaoning Provincial Key Laboratory of Cerebral Diseases, \\ Department of Physiology, Dalian Medical University, Dalian, Liaoning 116044, P.R. China
}

Received May 22, 2018; Accepted February 1, 2019

DOI: $10.3892 / \mathrm{ol} .2019 .10062$

\begin{abstract}
Bufalin (BF) is a cardiotonic steroid that has recently been found to have substantial anticancer activity; however, more efforts should be directed toward clarifying the detailed molecular mechanisms underlying this activity. BF could exert its anticancer effect by inducing apoptosis in various human cancer cells and thus triggering autophagic cancer cell death. The anti-inflammatory activities of BF are potentially important for its anticancer functions. Notably, some promising synthetic BF derivatives, including poly (ethylene glycol)-based polymeric prodrug of BF and BF211, have shown potent anticancer activity. Additionally, clinical trials regarding the use of BF-related agents in patients have supported the positive effect of BF as an anticancer treatment. Currently, large-scale randomized, double-blind, placebo or positive drug parallel controlled studies are required to confirm the anticancer potential of BF in various cancer types in the clinical setting. The present review will evaluate the potential mechanisms mediated by BF in intracellular signaling events in cancer cells and various promising BF derivatives that may have greater anticancer activity, thereby clarifying BF-mediated anticancer effects. The experimental and clinical results reviewed strongly emphasize the importance of this topic in future investigations.
\end{abstract}

Correspondence to: Dr Bo Zhang, Department of Neurosurgery, The Second Affiliated Hospital of Dalian Medical University, 467 Zhong Shan Road, Dalian, Liaoning 116023, P.R. China

E-mail: zhangbodl@126.com

Dr Shao Li, Liaoning Provincial Key Laboratory of Cerebral Diseases, Department of Physiology, Dalian Medical University, 9 Western Section, Lvshun South Street, Dalian, Liaoning 116044, P.R. China

E-mail: lishao89@dmu.edu.cn

${ }^{*}$ Contributed equally

Key words: bufalin, Chansu, cancer, mechanism, treatment

\section{Contents}

1. Introduction

2. BF could exert antitumor activity by inducing the apoptosis of various human cancer cells

3. BF could exert anticancer effects by triggering autophagic cancer cell death

4. BF could exert anticancer effects via its anti-inflammatory activity

5. Promising BF derivatives may have even greater anticancer activity

6. Clinical trials regarding the use of $\mathrm{BF}$ in patients

7. Conclusion

\section{Introduction}

Bufalin (BF) is a cardiotonic steroid isolated from the Chinese toad venom, Chansu, a galenical preparation of the dried white venom of Chinese Bufo gargarizans (Asiatic toad) $(1,2)$, with a molecular formula of $\mathrm{C}_{24} \mathrm{H}_{34} \mathrm{O}_{4}$ and a relative molecular weight of $386.5 \mathrm{~g} / \mathrm{mol}$. As an active compound extracted from a Chinese traditional medicine, BF exerts various biological effects, including pain relief, myocardial contraction stimulation, blood pressure stimulation, anti-inflammatory and antineoplastic activities (3-5). Since 2010, BF has received increased attention due to its anticancer effects on a wide range of cancer types (e.g., lung, liver, prostate, gastric, colon and gastric cancer) (6-12). This compound could mediate cell cycle arrest, cell growth inhibition, apoptosis and the expression of genes associated with the malignant phenotype in human cancer cells (13). In addition, BF can be used safely for an extended period without marked side effects $(12,13)$. Furthermore, transformed cells could be more susceptible to the effects of BF than normal cells (13). All of these factors have prompted the present review to analyze the potential of BF in anticancer treatments. However, the precise molecular mechanisms by which BF induces tumor suppression remain unclear. Microarray analysis revealed possible target-related proteins and genes of BF in cancer cells (11). A proteomic-based study was performed by Xie et al (11), and following BF treatment, 
24 differentially expressed proteins were identified using a comparative proteomics approach. The study found that the downregulation of heat shock protein 27 (Hsp27) could serve a critical role in BF-induced apoptosis in osteosarcoma cells. Subsequently, Zhang et al (14) explored target-related proteins using two quantitative proteomic methods (isobaric tags for relative and absolute quantitation-based and label-free proteomic analysis) in lung cancer. These two proteomic methods were complementary, and suggested that oxidative stress and regulation of relevant gene expression were significantly involved in the effects of $\mathrm{BF}$, while the fibronectin-associated pathway was found to be important. Bioinformatics analysis revealed that the fibronectin-associated pathway is the most distinct pathway in the signal network of BF, and BF-induced protein expression changes, including decreased expression of fibronectin, increased expression of paxillin, calpain 2 and cell division control protein 42 homolog, , have been further confirmed in the fibronectin-associated pathway using immunoblotting (14). In addition, the genetic mechanisms underlying BF-induced DNA damage and apoptosis in lung cancer cells have been further elucidated (15). Wu et al (15) demonstrated that numerous genes associated with cell cycle regulation, apoptosis and DNA repair are significantly altered following BF treatment. Analysis of these gene alterations by Wu et al (15) and Zhang et al (14) could aid the elucidation of the mechanism underlying the cytotoxicity of BF at the genetic level and potentially offer various biomarkers for the treatment and diagnosis of lung cancer. Certainly, further studies are required to improve the understanding of how BF suppresses cancerous cells and does not affect normal cells. Furthermore, combinations of BF with cytotoxic agents, differentiation-inducing agents and even gene therapy may represent potential novel therapeutic strategies for cancer treatment. The present review aims to evaluate the anticancer properties of $\mathrm{BF}$ from the perspective of emerging treatment options for cancer patients.

\section{BF could exert antitumor activity by inducing the apoptosis of various human cancer cells}

Inducing apoptosis in target cells could be a key mechanism for the majority of anticancer therapies. BF is a cardiotonic steroid that has the potential to induce cancer cell apoptosis (12). Cell apoptosis was induced in human non-small cell lung cancer A549 cells following treatment with BF, while suppression of cell proliferation occurred in a time- and dose-dependent manner, and induced cell cycle arrest at the $\mathrm{G}_{1}$ phase was found (6). Li et al (7) identified that BF exerts antitumor effects by triggering apoptosis and inducing cell cycle arrest in pancreatic cancer cells. Notably, in pancreatic cancer cells, BF could also promote the growth inhibition effect of gemcitabine (7). For the first time, Jiang et al (16) indicated that BF could be a potential therapy for treating gallbladder cancer. The study demonstrated that $\mathrm{BF}$ induces cell cycle arrest and apoptosis in gallbladder carcinoma cells. Treatment of human bladder carcinoma T24 cells with BF had a significant growth inhibition effect $(\mathrm{P}<0.05)$, compared with vehicle treatment. This effect is likely attributable to the prominent arrest of cancer cells in the $G_{2} / M$ phase of the cell cycle and the apoptosis stimulated by BF (17), as evidenced by formation of apoptotic bodies, chromatin condensation and cell accumulation in the sub- $\mathrm{G}_{1}$ phase. In breast cancer, $B F$ greatly sensitized estrogen receptor (ER) $\alpha$-positive MCF-7 and ER $\alpha$-negative MDA-MB-231 human breast cancer cells to tumor necrosis factor (TNF)-related apoptosis-inducing ligand (TRAIL)-induced apoptosis (18), compared with vehicle treatment. Notably, BF increases TRAIL-induced apoptosis from $2.0 \pm 0.5$ to $30.1 \pm 1.2 \%$ in MCF-7 cells, and from $6.9 \pm 1.8$ to $41.5 \pm 1.4 \%$ in MDA-MB-231 cells, which indicated that MCF-7 cells are more sensitive to BF than MDA-MB-231 cells. The enhanced apoptotic effects of the TRAIL/BF combination were also associated with the augmentation of caspase activation (18). In leukemia, BF could exert strong differentiation-inducing activity in three human leukemia-derived cell lines (myeloblastic ML1, human promyelocytic HL60 and monoblastic U937) at a concentration of $10 \mathrm{nM}$. However, treatment of human leukemia K562 cells with other cardiotonic steroids, including digitoxigenin, cinobufagin and ouabain, at the same concentration only had a weak or no effect on these cells $(19,20)$. These findings indicate that BF may have potential in human myelogenous leukemia differentiation therapy (19-21). Notably, Jing et al (22) demonstrated that in normal polymorphonuclear and mononuclear cells, apoptotic cell death was not induced by $\mathrm{BF}$, indicating that the anticancer effects of BF may be cell-type specific. In gastric cancer, $\mathrm{BF}$ could inhibit the proliferation of gastric cancer MGC803 cells by inducing apoptosis, and the phosphatidylinositol 3-kinase/protein kinase B (Akt) pathway may serve a key role in this process (23). The antiproliferative and apoptosis-inducing mechanisms of $\mathrm{BF}$ in prostate cancer cells have also been investigated $(24,25)$. In hepatocellular carcinoma, BF exhibited significant anticancer effects on the orthotopic transplantation tumor model of human hepatocellular carcinoma in nude mice and could promote the apoptosis of transplanted tumor cells with no marked toxicity (26). In our previous study investigating glioma (27), it was demonstrated that BF inhibits glioma growth by promoting proteasomal degradation of the sodium/potassium-adenosine 5'-triphosphatase $\alpha$-1 subunit (ATP1A1), and ATP1A1 deficiency could inhibit the proliferation of glioma via promotion of apoptosis. Notably, BF was revealed to penetrate the blood-brain barrier (BBB) (27). Rats were intraperitoneally injected with $\mathrm{BF}$ and cerebrospinal fluid (CSF) was collected from the cerebello-medullary cistern by penetrating the foramen magnum. The content of BF in the CSF was detected using liquid chromatography/mass spectrometry assays to prove that BF could traverse the BBB (27). Therefore, we hypothesize that $\mathrm{BF}$ can enter the brain via the $\mathrm{BBB}$ and thus exert a more powerful effect on tumors in the central nervous system.

\section{BF could exert anticancer effects by triggering autophagic cancer cell death}

Apoptosis, necrosis and autophagic cell death are the three major morphological processes responsible for cell death $(28,29)$. Autophagy is an important cellular catabolic process that maintains homeostasis by degrading dysfunctional cellular organelles and excessive proteins in living cells (30). The cytoplasms and double smooth membranes (phagophores) of numerous types of organelles, including the endoplasmic reticulum, peroxisomes and mitochondria, can form autophagosomes. The autophagosome then fuses with the lysosome to form autophagolysosomes, finally leading to 
degradation of the captured proteins or organelles by lysosomal enzymes (31-33). Promoting autophagic cell death is an important strategy for the chemotherapeutic treatment of cancer (34-36). Xie et al (10) demonstrated that BF could induce autophagic cell death via c-Jun $\mathrm{N}$-terminal kinase activation and the generation of reactive oxygen species in human colon cancer HT29 cells. Tsai et al (37) also found that in SK-HEP-1 cells, BF induced autophagic cell death and cell cycle arrest via the Akt/mechanistic target of rapamycin signaling pathway. These findings indicate that BF may be a possible treatment of human hepatocellular carcinoma. Notably, Shen et al (38) explored the potential of BF in glioma for the first time. The study found that preventing autophagy promoted apoptosis and increased the induction of ER stress-associated proteins, indicating that autophagy could exert a cytoprotective effect on cell death and ER stress induced by BF. This result indicates that BF could inhibit glioma cell growth and induce interplay between autophagy and apoptosis via ER stress, and more importantly, provides a molecular basis for developing $\mathrm{BF}$ into a drug candidate for glioma treatment. The amount of research on the association between autophagy and cancer has increased rapidly. Cancer cells may utilize autophagy to enhance their survival in the hostile tumor microenvironment with an altered metabolism, indicating that suppression of autophagy is required from therapeutic cancer treatment strategies (39). Indeed, the interaction between autophagy and apoptosis, which depends on the use of chemotherapeutic drugs, as well as the type of cancer cell, could significantly impact the fate of human cancer cells.

\section{BF could exert anticancer effects via its anti-inflammatory activity}

Chansu has been used in the treatment of inflammatory diseases in China for thousands of years (40); however, little is known about the anti-inflammatory mechanisms of BF. Nuclear factor $-\kappa B(N F-\kappa B)$ is a central regulator of the inflammatory process and could regulate a group of proinflammatory mediators, including inducible nitric oxide synthase, cyclooxygenase-2, interleukin (IL)-1 $\beta$, TNF and IL-6 (41). In 2011, Ye et al (42) suggested that BF suppresses the nuclear translocation of $N F-\kappa B$ in response to TNF in human kidney tissue cells. BF was also shown to modulate $N F-\kappa B$ activity in human osteosarcomas in a previous study (11). NF- $\kappa \mathrm{B}$ signaling could be a potential therapeutic target of BF for the pathogenesis of inflammation and cancer. Wen et al (43) demonstrated that compared with vehicle treatment, BF exerts a strong anti-inflammatory effect on carrageenan-induced paw edema in rats, which is a commonly used model for the investigation of inflammation. The study demonstrated that $\mathrm{BF}$ significantly $(\mathrm{P}<0.05)$ suppressed the activation of $\mathrm{NF}-\kappa \mathrm{B}$ in vivo by maintaining I $\mathrm{B} \alpha$ levels and inhibiting the nuclear translocation of NF- $\kappa \mathrm{B}$ p 65. Furthermore, the downstream $\mathrm{NF}-\kappa \mathrm{B}$ proinflammatory mediators, cyclooxygenase-2, inducible nitric oxide synthase, TNF- $\alpha$, IL- 6 and IL-1 $\beta$ were also suppressed. Therefore, BF could possess strong in vivo anti-inflammatory activity, thus serving a role in reducing $\mathrm{NF}-\kappa \mathrm{B}$ activation and the inhibition of downstream proinflammatory mediators. Inflammatory pathways have been targeted in attempts to treat cancer (44-46), and the association between cancer and inflammation has returned to the forefront of clinical oncology. These findings support BF as a potential novel therapeutic agent for alleviating inflammation, and more importantly, for treating various cancer types.

\section{Promising BF derivatives may have even greater anticancer activity}

While research into BF has revealed its potential in the treatment of different human cancer types, further investigation is required prior to its use as a cancer treatment. BF has a narrow therapeutic window, extremely low water solubility, unsatisfactory bioavailability and severe adverse effects, including high cardiac toxicity, which limits its clinical applications $(47,48)$. Since the purification and total synthesis of BF, various methods have been used to improve its activity and expand its therapeutic potential in different biological systems by modifying its structure (49,50). More importantly, efforts should be directed towards identifying additional $\mathrm{BF}$ derivatives that exert stronger anticancer effects and have a lower toxicity level compared with the natural compound to promote the development of novel anticancer agents from cardiac steroids, including BF.

Notably, Liu et al (51) synthesized a novel polymeric prodrug of $\mathrm{BF}$, poly (ethylene glycol)-based polymeric prodrug of BF (PEGS-BF). The water solubility and stability of PEGS-BF were improved without loss of its anticancer activity compared with BF. In addition, in vitro and in vivo experiments showed that PEGS-BF exerted anticancer effects comparable to those of unbound BF. The reported improved stability, water solubility and controlled drug release features of this polymeric prodrug provide strong arguments for its use in potential clinical applications.

BF211 is a BF derivative with a stronger cytotoxic activity than BF in cancer cells $(52,53)$. In nude immunodeficient Balb/c-nu-nu mice inoculated with A549 cells, BF211 also exhibited significantly $(\mathrm{P}<0.05)$ stronger suppressive effects on tumor growth compared with BF (53). Notably, as the acute toxicity of BF211 was lower compared with that of BF [lethal dose $50\left(\mathrm{LD}_{50}\right)$ value of $\mathrm{BF} 211$ was $14.75 \mathrm{mg} / \mathrm{kg}$ for male mice and $18.21 \mathrm{mg} / \mathrm{kg}$ for female mice, whereas $\mathrm{LD}_{50}$ value of $\mathrm{BF}$ in mice was $2.2 \mathrm{mg} / \mathrm{kg}], \mathrm{BF} 211$ could be used at higher concentrations for cancer treatment, indicating that BF211 could have a wider therapeutic window. In summary, studies by Liu et al (53) demonstrated that BF211 is a potential novel anticancer compound with a relatively lower toxicity compared with $\mathrm{BF}$ as aforementioned $\left(\mathrm{BF} 211 \mathrm{LD}_{50}\right.$ value

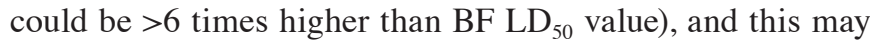
be due to its specific binding characteristics for sodium-potassium adenosine triphosphatase. Furthermore, Sun et al (54) attempted to identify target-related proteins of BF211 in A549 cells to further elucidate the mechanism underlying its anticancer effects. Their findings suggested that BF211 may impact multiple cellular functions, including translation, transcription and protein synthesis. In addition, a previous study (54) also explored the effect of BF211 on regulating proteasome activities and revealed only moderate inhibitory effects; thus, there is currently no direct evidence that the anticancer effects of BF211 are mediated by proteasome inhibition and it is possible that the contribution of proteasome inhibition to the cytotoxicity of BF211 is minimal. 


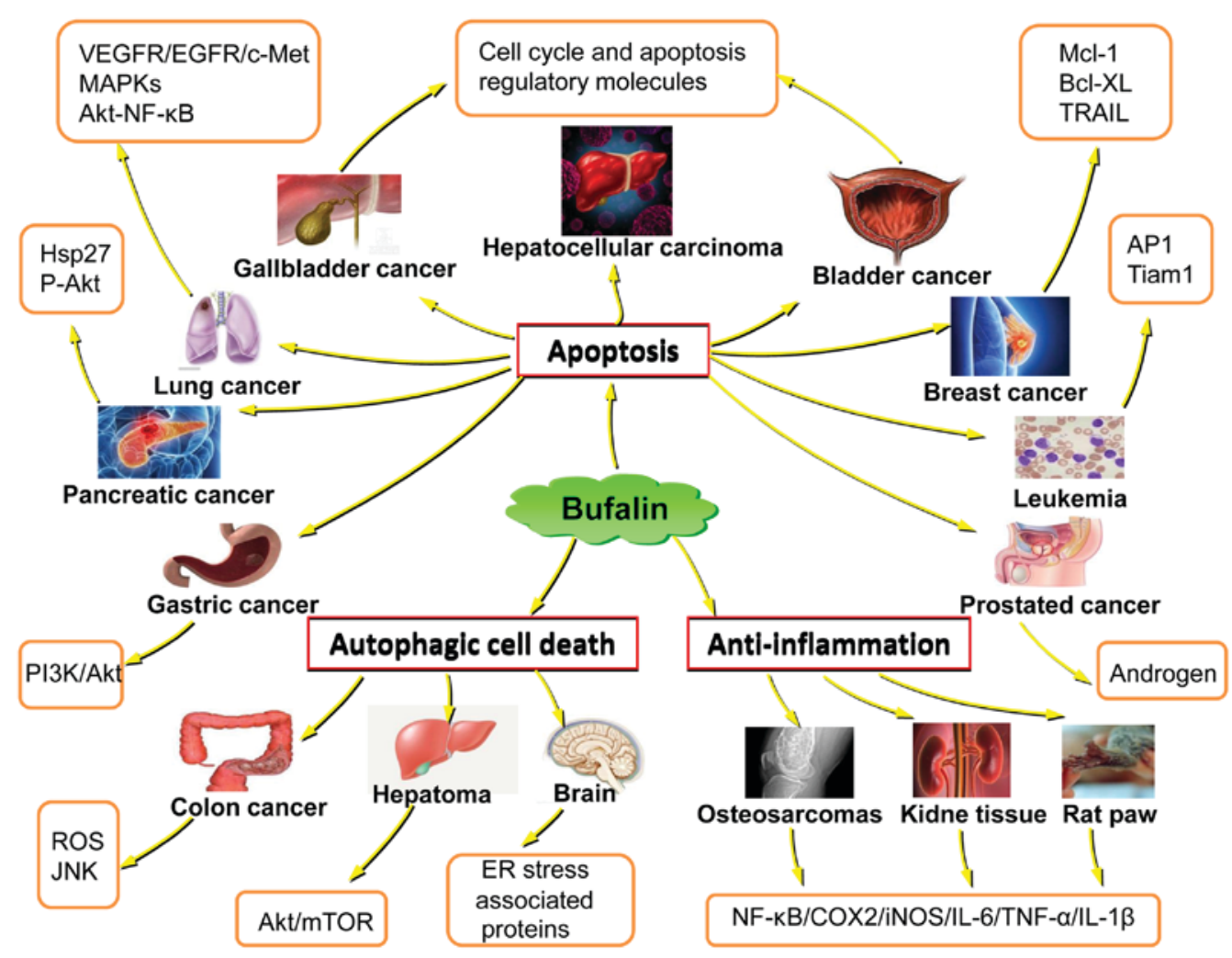

Figure 1. Schematic diagram displaying the anticancer properties of bufalin, from the perspective of the emerging treatment targets for various cancer types. Hsp27, heat shock protein 27; P-Akt, phosphorylated protein kinase B; VEGFR, vascular endothelial growth factor receptor; EGFR, epidermal growth factor

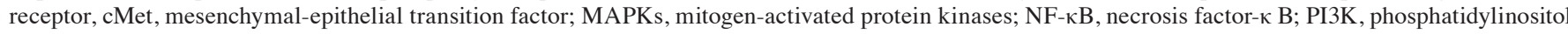
3-kinase; ROS, reactive oxygen species; JNK, c-Jun N-terminal kinases; mTOR, mechanistic target or rapamycin; ER, endoplasmic reticulum; COX2, cyclooxygenase 2; iNOS, inducible nitric oxide synthase; IL, interleukin; TNF- $\alpha$, tumor necrosis factor- $\alpha$; AP1, activating protein 1; Tiam1, T-lymphoma invasion and metastasis-inducing protein; Mcl-1, induced myeloid leukemia cell differentiation protein; Bcl-XL, B-cell lymphoma-extra large; TRAIL, tumor necrosis factor-related apoptosis-inducing ligand.

\section{Clinical trials regarding the use of $\mathrm{BF}$ in patients}

Chansu, the galenical preparation of the dried white venom of Chinese Bufo gargarizans, has been widely used for the treatment of cancer at oncology clinics in China (55). Huachansu, one of the main biologically active components of BF, is a Chinese medicine derived from dried toad venom from the skin glands of Bufo bufo gargarizans Cantor or Bufo melanotictus Schneider (55). Huachansu is manufactured by Anhui Jinchan Biochemistry Company Ltd., in Huaibei, China (Chinese FDA no. ISO9002) and includes 2 primary biologically active chemical components: Indole alkaloids (bufotenidine, bufotenine, serotonin and cinobufotenine) and steroidal cardiac glycosides ( $>28$ have been identified, including BF, cinobufagin, resibufogenin, marinobufagin, cinobufotalin and bufotalin) (55). A previous study confirmed that BF, cinobufagin and resibufogenin are the 3 major cardiac glycosides, which the anticancer activity of Huachansu can be attributed to (56). A pilot study investigating the use of Huachansu in patients with advanced cancer was performed by Meng et al (57), using a phase I trial design. Huachansu was intravenously administered for 2 weeks followed by 1 week off, for each cycle. Without significant adverse events or progressive disease, treatment continued beyond two cycles. A total of 15 patients (11 with hepatocellular cancer, 2 with pancreatic cancer and 2 with non-small cell lung cancer) were included in the trial. Overall 6 patients $(40 \%)$ had stable disease (median duration, 6.0 months; range, 3.5-11.1 months). In addition, 1 patient with hepatocellular cancer had a $20 \%$ reduction in tumor mass (duration of disease stability in response to Huachansu alone, 11 months). The plasma BF concentration reached maximal levels at the end of the 2-h infusion and was proportional to the amount of drug administered. Notably, a dose-dependent increase in BF levels was observed in all the patients, with no evidence of drug accumulation in the plasma, which may have been due to the short half-life of the drug. Although there was no correlation between the plasma BF level and the anticancer effect of Huachansu, the small number of patients studied precluded drawing an association between the drug dose and response. One limitation of the study by Meng et al (57) was the absence of a control group. Significantly, Huachansu has been found to be well tolerated, even at doses eight times those normally administered (normally administered doses: Level 1, $10 \mathrm{ml} / \mathrm{m}^{2}$; level 2, $20 \mathrm{ml} / \mathrm{m}^{2}$; level 3, $40 \mathrm{ml} / \mathrm{m}^{2}$; level 4, $60 \mathrm{ml} / \mathrm{m}^{2}$; and level $5,90 \mathrm{ml} / \mathrm{m}^{2}$ ) in China, and can lead to disease stabilization in certain patients according to the results of a phase I clinical trial (57).

\section{Conclusion}

In summary, the potential roles of $\mathrm{BF}$ in various cancer types have been increasingly recognized, but the specific mechanisms have not been fully clarified (Fig. 1). The present review demonstrates that $\mathrm{BF}$ has marked antitumor activity. BF could exert an antitumor effect by inducing apoptosis and triggering autophagic cell death in various human cancer cells. 
The anti-inflammatory activities of BF are also important for its antitumor function. Notably, an increasing number of BF derivatives have shown potent anticancer activity, including PEGS-BF and BF211. Clinical trials regarding the use of BF in cancer patients have supported the positive effect of BF on cancer treatment.

An increasing number of clinical studies will focus on investigating the use of $\mathrm{BF}$ in cancer treatment in the near future. Research has revealed that BF has potential as a potent, novel and effective anticancer therapy for cancer patients. However, more BF derivatives with stronger anticancer effects and lower toxicity levels should be identified to promote the development of additional novel anticancer agents. Furthermore, large-scale randomized studies are required to investigate the efficacy of BF in various cancer types. This review provides critical information for the design of larger and more focused clinical studies that are necessary to systematically and definitively evaluate the role of BF in cancer treatments.

\section{Acknowledgements}

Not applicable.

\section{Funding}

This study is supported by grants from the National Natural Science Foundation of China (nos. 81372714 and 81672480), the Liaoning Provincial Natural Science Foundation of China (nos. 201602244 and 2016010217-30), the Distinguished Professor Project of Liaoning Province, the Special Grant for Translational Medicine, the Dalian Medical University (no. 2015002) and the Basic research projects in colleges and universities of Liaoning Province (no. LQ2017033).

\section{Availability data and materials}

Not applicable.

\section{Author contributions}

YL, JL, XJ, XW, JX, SL and BZ contributed in the design and writing of the review. All authors read and approved the final manuscript.

\section{Ethics approval and consent to participate}

Not applicable.

\section{Patient consent for publication}

Not applicable.

\section{Competing interests}

The authors declare that they have no competing interests.

\section{References}

1. Wang J, Xia Y,Zuo Q and Chen T: Molecular mechanisms underlying the antimetastatic activity of bufalin. Mol Clin Oncol 8 631-636, 2018.
2. Dai XY, Zhou BF, Xie YY, Lou J and Li KQ: Bufalin and 5-fluorouracil synergistically induce apoptosis in colorectal cancer cells. Oncol Lett 15: 8019-8026, 2018.

3. Cui X, Inagaki Y, Xu H, Wang D, Qi F, Kokudo N, Fang D and Tang W: Anti-hepatitis B virus activities of cinobufacini and its active components bufalin and cinobufagin in HepG2.2.15 cells. Biol Pharm Bull 33: 1728-1732, 2010.

4. Lu CX, Nan KJ and Lei Y: Agents from amphibians with anticancer properties. Anticancer Drugs 19: 931-939, 2008.

5. Qi F, Li A, Inagaki Y, Kokudo N, Tamura S, Nakata M and Tang W: Antitumor activity of extracts and compounds from the skin of the toad Bufo bufo gargarizans Cantor. Int Immunopharmacol 11: 342-349, 2011.

6. Jiang Y, Zhang Y, Luan J, Duan H, Zhang F, Yagasaki K and Zhang G: Effects of bufalin on the proliferation of human lung cancer cells and its molecular mechanisms of action. Cytotechnology 62: 573-583, 2010

7. Li M, Yu X, Guo H, Sun L, Wang A, Liu Q, Wang X and Li J: Bufalin exerts antitumor effects by inducing cell cycle arrest and triggering apoptosis in pancreatic cancer cells. Tumor Biol 35: 2461-2471, 2014

8. Qiu DZ, Zhang ZJ, Wu WZ and Yang YK: Bufalin, a component in Chansu, inhibits proliferation and invasion of hepatocellular carcinoma cells. BMC Complement Altern Med 13: 185-195, 2013.

9. Qiu YY, Hu Q, Tang QF, Feng W, Hu SJ, Liang B, Peng W and Yin PH: MicroRNA-497 and bufalin act synergistically to inhibit colorectal cancer metastasis. Tumor Biol 35: 2599-2606, 2014.

10. Xie CM, Chan WY, Yu S, Zhao J and Cheng CH: Bufalin induces autophagy-mediated cell death in human colon cancer cells through reactive oxygen species generation and JNK activation. Free Radic Biol Med 51: 1365-1375, 2011.

11. Xie XB, Yin JQ, Wen LL, Gao ZH, Zou CY, Wang J, Huang G, Tang QL, Colombo C, He WL, Jia Q and Shen JN: Critical role of heat shock protein 27 in bufalin-induced apoptosis in human osteosarcomas: A proteomic-based research. PLoS One 7: e47375, 2012.

12. Yin PH, Liu X, Qiu YY, Cai JF, Qin JM, Zhu HR and Li Q: Anti-tumor activity and apoptosis-regulation mechanisms of bufalin in various cancers: New hope for cancer patients. Asian Pac J Cancer Prev 13: 5339-5343, 2012.

13. Takai N, Kira N, Ishii T, Yoshida T, Nishida M, Nishida Y, Nasu K and Narahara H. Bufalin, a traditional oriental medicine, induces apoptosis in human cancer cells. Asian Pac J Cancer Prev 13: 399-402, 2012.

14. Zhang DM, Feng LX, Liu M, Jin WH, Luo J, Nie AY, Zhou Y, Li Y, Wu WY, Jiang BH, et al: Possible target-related proteins and signal network of bufalin in A549 cells suggested by both iTRAQ-based and label-free proteomic analysis. Proteomics 16: 935-945, 2016.

15. Wu SH, Hsiao YT, Chen JC, Lin JH, Hsu SC, Hsia TC, Yang ST, Hsu WH and Chung JG: Bufalin alters gene expressions associated DNA damage, cell cycle and apoptosis in human lung cancer NCI-H460 cells in vitro. Molecules 19: 6047-6057, 2014.

16. Jiang L, Zhao MN, Liu TY, Wu XS, Weng H, Ding Q, Shu YJ, Bao RF, Li ML, Mu JS, et al: Bufalin induces cell cycle arrest and apoptosis in gallbladder carcinoma cells. Tumour Biol 35: 10931-10941, 2014.

17. Hong SH and Choi YH: Bufalin induces apoptosis through activation of both the intrinsic and extrinsic pathways in human bladder cancer cells. Oncol Rep 27: 114-120, 2012.

18. Dong Y, Yin S, Li J, Jiang C, Ye M and Hu H: Bufadienolide compounds sensitize human breast cancer cells to TRAIL-induced apoptosis via inhibition of STAT3/Mcl-1 pathway. Apoptosis 16: 394-403, 2011.

19. Zhu Z, Li E, Liu Y, Gao Y, Sun H, Wang Y, Wang Z, Liu X, Wang Q and Liu Y: Bufalin induces the apoptosis of acute promyelocytic leukemia cells via the downregulation of survivin expression. Acta Haematol 128: 144-150, 2012.

20. Zhai X, Lu J, Wang Y, Fang F, Li B and Gu W: Reversal effect of bufalin on multidrug resistance in K562/VCR vincristine-resistant leukemia cell line. J Tradit Chin Med 34: 678-683, 2014.

21. Wang LP, Zhao YN, Sun X and Gao RL: Effects of bufalin on up-regulating methylation of Wilm's tumor 1 gene in human erythroid leukemic cells. Chin J Integr Med 23: 288-294, 2017.

22. Jing Y, Watabe M, Hashimoto S, Nakajo S and Nakaya K: Cell cycle arrest and protein kinase modulating effect of bufalin on human leukemia ML1 cells. Anticancer Res 14: 1193-1198, 1994.

23. Li D, Qu X, Hou K, Zhang Y, Dong Q, Teng Y, Zhang J and Liu Y: PI3K/Akt is involved in bufalin-induced apoptosis in gastric cancer cells. Anticancer Drugs 20: 59-64, 2009. 
24. Yeh JY, Huang WJ, Kan SF and Wang PS: Effects of bufalin and cinobufagin on the proliferation of androgen dependen and independent prostate cancer cells. Prostate 54: 112-124, 2003.

25. Yu CH, Kan SF, Pu HF, Jea Chien E and Wang PS: Apoptotic signaling in bufalin- and cinobufagin-treated androgen-dependent and-independent human prostate cancer cells. Cancer Sci 99: 2467-2476, 2008.

26. Han KQ, Huang G, Gu W, Su YH, Huang XQ and Ling CQ Anti-tumor activities and apoptosis-regulated mechanisms of bufalin on the orthotopic transplantation tumor model of human hepatocellular carcinoma in nude mice. World J Gastroenterol 13: 3374-3379, 2007

27. Lan YL, Wang X, Lou JC, Xing JS, Yu ZL, Wang H, Zou S, Ma $X$ and Zhang B: Bufalin inhibits glioblastoma growth by promoting proteasomal degradation of the $\mathrm{Na}^{+} / \mathrm{K}^{+}$-ATPase $\alpha 1$ subunit. Biomed Pharmacother 103: 204-215, 2018.

28. Choi KS: Autophagy and cancer. Exp Mol Med 44: 109-120, 2012.

29. Naumann P, Fortunato F, Zentgraf H, Büchler MW, Herr I and Werner J: Autophagy and cell death signaling following dietary sulforaphane act independently of each other and require oxidative stress in pancreatic cancer. Int J Oncol 39: 101-109, 2011.

30. Rabinowitz JD and White E: Autophagy and metabolism. Science 330: 1344-1348, 2010

31. Lee J, Giordano S and Zhang J: Autophagy, mitochondria and oxidative stress: Cross-talk and redox signalling. Biochem J 441: 523-540, 2012

32. Martinez-Borra J and Lopez-Larrea C: Autophagy and self-defense. Adv Exp Med Biol 738: 169-184, 2012.

33. Anders HJ and Schlondorff DO: Innate immune receptors and autophagy: Implications for autoimmune kidney injury. Kidney Int 78: 29-37, 2010.

34. Ropolo A, Bagnes CI, Molejon MI,Lo Re A, Boggio V, Gonzalez CD and Vaccaro MI: Chemotherapy and autophagy-mediated cell death in pancreatic cancer cells. Pancreatology 12: 1-7, 2012.

35. Guo XL, Li D, Hu F, Song JR, Zhang SS, Deng WJ, Sun K, Zhao QD, Xie XQ, Song YJ, et al: Targeting autophagy potentiates chemotherapy-induced apoptosis and proliferation inhibition in hepatocarcinoma cells. Cancer Lett 320: 171-179, 2012

36. Yousefi S and Simon HU: Autophagy in cancer and chemotherapy. Results Probl Cell Differ 49: 183-190, 2009.

37. Tsai SC, Yang JS, Peng SF, Lu CC, Chiang JH, Chung JG, Lin MW, Lin JK, Amagaya S, Wai-Shan Chung C, et al: Bufalin increases sensitivity to AKT/mTOR-induced autophagic cell death in SK-HEP-1 human hepatocellular carcinoma cells. Int J Oncol 41: 1431-1442, 2012.

38. Shen S, Zhang Y, Wang Z, Liu R and Gong X: Bufalin induces the interplay between apoptosis and autophagy in glioma cells through endoplasmic reticulum stress. Int J Biol Sci 10: 212-224, 2014.

39. Tsujimoto Y and Shimizu S: Another way to die: Autophagic programmed cell death. Cell Death Differ 12 (Suppl 2): S1528-S1534, 2005.

40. Chen KK and Kovaríková A: Pharmacology and toxicology of toad venom. J Pharm Sci 56: 1535-1541, 1967.

41. Rahman A and Fazal F: Blocking NF- $\kappa$ B: An inflammatory issue. Proc Am Thorac Soc 8: 497-503, 2011.

42. Ye J, Chen S and Maniatis T: Cardiac glycosides are potent inhibitors of interferon- $\beta$ gene expression. Nat Chem Biol 7 : 25-33, 2011.
43. Wen L, Huang Y, Xie X, Huang W, Yin J, Lin W, Jia Q and Zeng W: Anti-inflammatory and antinociceptive activities of bufalin in rodents. Mediators Inflamm 2014: 171839, 2014.

44. Colombo MP and Mantovani A: Targeting myelomonocytic cells to revert inflammation-dependent cancer promotion. Cancer Res 65: 9113-9116, 2005.

45. Huang Y, Snuderl M and Jain RK: Polarization of tumor-associated macrophages: A novel strategy for vascular normalization and antitumor immunity. Cancer Cell 19: 1-2, 2011

46. Huang Y, Yuan J, Righi E, Kamoun WS, Ancukiewicz M, Nezivar J, Santosuosso M, Martin JD, Martin MR, Vianello F, et al: Vascular normalizing doses of antiangiogenic treatment reprogram the immunosuppressive tumor microenvironment and enhance immunotherapy. Proc Natl Acad Sci USA 109: 17561-17566, 2012

47. Hu Q, Liang B, Sun Y, Guo XL, Bao YJ, Xie DH, Zhou M, Duan YR, Yin PH and Peng ZH: Preparation of bufalin-loaded pluronic polyetherimide nanoparticles, cellular uptake, distribution, and effect on colorectal cancer. Int J Nanomedicine 9: 4035-4041, 2014

48. Yang Z, Teng Y, Wang $\mathrm{H}$ and Hou $\mathrm{H}$ : Enhancement of skin permeation of bufalin by limonene via reservoir type transdermal patch: Formulation design and biopharmaceutical evaluation. Int J Pharm 447: 231-240, 2013.

49. Pettit GR, Houghton LE, Knight JC and Bruschwe F: Synthesis of bufalin. J Chem Soc D Chem Commun 2: 93-94, 1970.

50. Yuan XF, Tian HY, Li J, Jin L, Jiang ST, Liu KW, Luo C, Middleton DA, Esmann M, Ye WC and Jiang RW: Synthesis of bufalin derivatives with inhibitory activity against prostate cancer cells. Nat Prod Res 28: 843-847, 2014.

51. Liu T, Yuan X, Jia T, Liu C, Ni Z, Qin Z and Yuan Y: Polymeric prodrug of bufalin for increasing solubility and stability: Synthesis and anticancer study in vitro and in vivo. Int J Pharm 506: 382-393, 2016.

52. Lei M, Xiao Z, Ma B, Chen Y, Liu M, Liu J, Guo D, Liu X and $\mathrm{Hu} \mathrm{L}$ : Synthesis and biological evaluation of bufalin-3-yl nitrogen-containing-carbamate derivatives as anticancer agents. Steroids 108: 56-60, 2016

53. Liu M, Feng LX, Sun P, Liu W, Wu WY, Jiang BH, Yang M, Hu LH, Guo DA and Liu X: A novel bufalin derivative exhibited stronger apoptosis-inducing effect than bufalin in A549 lung cancer cells and lower acute toxicity in mice. PLoS One 11: e0159789, 2016.

54. Sun P, Feng LX, Zhang DM, Liu M, Liu W, Mi T, Wu WY, Jiang BH, Yang M, Hu LH, et al: Bufalin derivative BF211 inhibits proteasome activity in human lung cancer cells in vitro by inhibiting $\beta 1$ subunit expression and disrupting proteasome assembly. Acta Pharmacol Sin 37: 908-918, 2016.

55. $\mathrm{Su} \mathrm{YH}$ and $\mathrm{Nu} \mathrm{X}$ : Evaluation of pharmacodynamic effect of pharmaceutical agents of Chan Su. J Beijing Univ TCM 24 51-54, 2001.

56. Su YH, Huang XQ, Zhang DZ, Zhang YN, Xie JM and Linh CQ: HPLC separation and determination of bufadienolide in cinobufacini injection. Chin Trad Patent Med 25: 24-27, 2003.

57. Meng Z, Yang P, Shen Y, Bei W, Zhang Y, Ge Y, Newman RA, Cohen L, Liu L, Thornton B, et al: Pilot study of huachansu in patients with hepatocellular carcinoma, nonsmall-cell lung cancer, or pancreatic cancer. Cancer 115: 5309-5318, 2009. 